\title{
ARTIGOORIGINAL
}

\section{Sistemas de posicionamento indoor (IPS) para aplicações de realidade aumentada: uma avaliação da precisão}

\section{Indoor positioning systems (IPS) for augmented reality: an analysis of accuracy}

\author{
Fábio Britto de Carvalho Almeida ${ }^{1}$, Ingrid Winkler ${ }^{\mathscr{C}}, 1$, Valter de Senna ${ }^{\mathscr{C}, 1}$ \\ ${ }^{1}$ Centro Universitário SENAI CIMATEC/ Programa de Pós-Graduação em Modelagem Computacional e Tecnologia \\ Industrial (PPGMCTI) \\ *falmeida@fieb.org.br; ingrid.winkler@fieb.org.br; senna@fieb.org.br
}

Recebido: 29/10/2019. Revisado: 08/01/2020. Aceito: 23/03/2020.

\section{Resumo}

A tecnologia Realidade Aumentada (RA) possibilita enriquecer o mundo real com informações oriundas do mundo virtual, através de um dispositivo tecnológico que execute aplicações em tempo real. Porém, ainda que já venha sendo utilizada em diferentes setores, a RA ainda possui limitações que impedem que seja adotada na área industrial de maneira mais efetiva e abrangente. Entre os desafios que fazem com que a tecnologia ainda não esteja madura, estão limitações relacionadas à interação com o usuário, aos dispositivos de hardware (que precisam ser mais confortáveis) e às técnicas de reconhecimento e rastreamento. O objetivo deste estudo é avaliar a imprecisão gerada por dispositivos de localização indoor na medição de coordenadas virtuais para futura aplicação em RA no contexto industrial. Para alcançá-lo, foi realizado um estudo dos sistemas de posicionamento indoor (IPS) existentes e deles foram selecionados apenas os que atendiam à premissa de precisão desta pesquisa. Por fim, estes dispositivos foram submetidos a ensaios de precisão, estabilidade e propagação de erro. Os resultados demonstraram que as tecnologias baseadas em imagens e ultrassom, representados pelos dispositivos Hololens e Marvelmind respectivamente, apresentaram resultados dentro do intervalo esperado neste trabalho.

Palavras-Chave: Precisão; Realidade Aumentada; Sistemas de Posicionamento Indoor;

\section{Abstract}

The technology augmented reality (AR) enables to enrich the real world with information from the virtual world, through a technological device that runs applications in real time. However, RA technology, despite being used in different sectors, it still has limitations that prevent it from being entered into the industrial field more effectively and comprehensively. Barriers that lead to it include limitations related to user interaction, hardware devices (which need to be more comfortable), and recognition and tracking techniques. The aim of this study is to evaluate the imprecision generated by indoor positioning systems in the measurement of virtual coordinates for future RA application in the industrial context. For this, a review of the existing indoor positioning systems (IPS's) was carried out and only those that met the precision premise of this research were selected. The results demonstrated that the technologies based on images and ultrasonic, represented by the devices Hololens and Marvelmind respectively, presented results within the expected range in this work.

Keywords: Augmented Reality; Indoor Positioning System; Precision; 


\section{Introdução}

A tecnologia Realidade Aumentada (RA) possibilita enriquecer o mundo real com informações oriundas do mundo virtual, através de um dispositivo tecnológico que execute aplicações em tempo real.

Para Azuma et al. (2001), RA é o casamento preciso de objetos virtuais e reais. Ou seja, às cenas do ambiente real são acrescidas informações virtuais contextuais relevantes, como textos, gráficos, vídeos, sons, feedbacks hápticos e dados de localização (Carmigniani and Furht, 2011).

A RA é mais do que uma tecnologia de exibição simples. Ela também representa um novo tipo de interface natural de usuário (NUI), que funciona em tempo real, para interação humana com objetos e dispositivos digitais. Essa tecnologia depende de quatro tarefas básicas e distintas, a saber: em primeiro lugar a captura de cena, em que a realidade a ser aumentada é apreendida através de um dispositivo de captura de vídeo, como uma câmera ou um dispositivo transparente, como uma tela montada na cabeça; em segundo lugar há a identificação da cena, em que a realidade capturada é digitalizada para definição da posição exata onde o conteúdo virtual será incorporado (posição essa que pode ser identificada por marcadores visuais ou por tecnologias de rastreamento como GPS, sensores infravermelhos ou laser); em seguida é realizado o processamento de cena, em que à medida que a cena é claramente reconhecida e identificada, o conteúdo virtual correspondente é solicitado, geralmente da Internet ou de qualquer tipo de banco de dados; e finalmente ocorre a visualização de cena, momento em que o sistema RA produz uma imagem misturando a cena real com o conteúdo virtual (Glockner et al., 2014).

Nesse processo, para que o conteúdo virtual seja sobreposto respeitando as condições do ambiente no qual está imerso, os sensores do dispositivo tecnológico captam dados do mundo real, ou seja, para que as informações sejam posicionadas precisamente no local exato do ambiente real, respeitando condições como oclusão, posicionamento e perspectiva, é importante possuir informações sobre o ambiente.

Aplicações de RA têm sido utilizadas em várias áreas para enfrentar dificuldades de visualização em cada domínio específico, como entretenimento, educação, engenharia, medicina, automotiva, aeroespacial e militar (Rankohi and Waugh, 2013). No contexto industrial, foco deste estudo, sua utilização começou no início da década de 1990 , com o projeto de montagem de pacotes de fios da Boeing (Azuma, 1997).

Porém, a tecnologia RA, apesar de ser utilizada em diferentes setores, ainda possui limitações que impedem que seja inserida no campo industrial de maneira mais efetiva e abrangente Palmarini et al. (2018). Entre as limitações que levam a que ainda não esteja madura estão limitações relacionadas à interação com o usuário, aos próprios dispositivos de hardware (que precisam ser mais confortáveis), e às técnicas de reconhecimento e rastreamento Palmarini et al. (2018). Martinetti et al. (2019) complementa essa informação ratificando que a tecnologia RA consegue penetrar em vários segmentos de mercado, mas sem domínios industriais convincentes (Martinetti et al., 2019).

Rastreamento é o alinhamento, em tempo real, dos mundos real e virtual através de imagens adquiridas por uma câmera em movimento (Comport et al., 2006). Os principais desafios atuais na etapa de rastreamento são alta precisão ${ }^{1}$ e baixa latência a um custo computacional razoável.

Sanna and Manuri (2016) afirmam que o alinhamento exato de recursos virtuais é um desafio que precisa ser superado e que representa uma falha fundamental em vários aplicativos de RA. Nessa linha, os trabalhos que investigam aplicações de RA no contexto industrial têm revelado que a falta de precisão no rastreamento do usuário e/ou objetos dentro do ambiente operacional ainda é um problema comum, seja em ambientes internos (indoor) quanto externos (outdoor).

Em ambientes indoor o desafio de manter a precisão aumenta, uma vez que quando se eleva a área de cobertura os pontos de referência podem se deslocar de um lugar para outro, devido a movimentos estruturais (Alarifi et al., 2016).

Diversos Sistemas de Posicionamento Indoor (IPS) já têm sido utilizados nos mais variados contextos, como para detectar e monitorar itens ou prestar assistência a idosos ou pessoas com deficiência, entre outros (Alarifi et al., 2016). Entretanto, nas aplicações de RA indoor, em ambientes industriais, ainda que aspectos como hardware, plataformas e algoritmos para IPS estejam em constante evolução (Gu et al., 2009) (Correa et al., 2016), e diversas tecnologias de detecção como RFID, WiFi, sinais acústicos, Bluetooth etc. venham sendo utilizadas, (Correa et al., 2016, Alarifi et al., 2016), ainda persiste o desafio de se ter um rastreamento preciso e robusto o suficiente. E, no entanto, nos cenários industriais, uma boa precisão no posicionamento da informação é muito importante, já que a falta de precisão pode levar, por exemplo, a que dois componentes muitos próximos sejam confundidos ou, em cenários complexos (com variação de iluminação, componentes muito pequenos etc.), a que operários posicionem-se no local errado, com consequências danosas. Ong et al. (2008) corroboram essa informação afirmando que a precisão do rastreamento de usuários dentro do cenário e a localização de objetos são preocupações comuns e relevantes para aplicações de RA em contextos sérios.

A eficiência de soluções de RA, em alguns casos, está diretamente associada a atividade de tracking e localização de objetos, tornando-a um dos principais alvo de pesquisas em RA (Zhou et al., 2008). Pode-se citar como exemplo os trabalhos de Neges et al. (2017) e Lee et al. (2008) que mostram uma diminuição no tempo médio para localização de objetos utilizando RA em comparação com a realização dessa mesma atividade sem sistemas de RA.

Diante do exposto, observa-se uma necessidade de estudos que avaliem a estabilidade e precisão de tecnologias responsáveis por posicionamento indoor, uma vez que elas possibilitam o rastreamento de usuários

\footnotetext{
${ }^{1}$ No contexto desse trabalho, precisão é a distância entre a posição estimada e a posição real (Liu et al., 2007)
} 
e objetos dentro de um ambiente e podem ser integradas com dispositivos de RA para auxiliar na localização desses objetos. Dessa forma, este trabalho tem como objetivo avaliar a imprecisão gerada por dispositivos de localização indoor na medição de coordenadas virtuais para futura aplicação em RA no contexto industrial.

Este trabalho caracteriza-se como um estudo experimental exploratório, que tem como meta proporcionar maior familiaridade com o problema, e está organizado em quatro seções: além desta Introdução, a seção 2 descreve os aspectos metodológicos, a seção 3 descreve os resultados obtidos nos experimentos realizados e discute-os e, finalmente, na seção 4 são feitas as considerações finais e indicações de pesquisas futuras.

\section{Aspectos metodológicos}

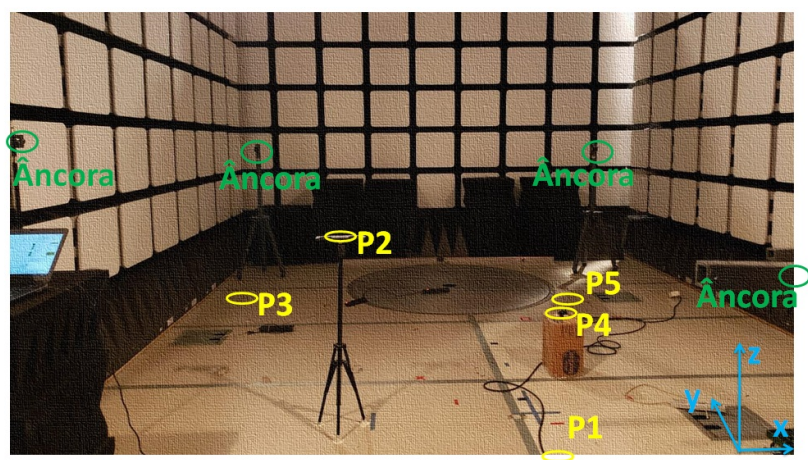

(a)

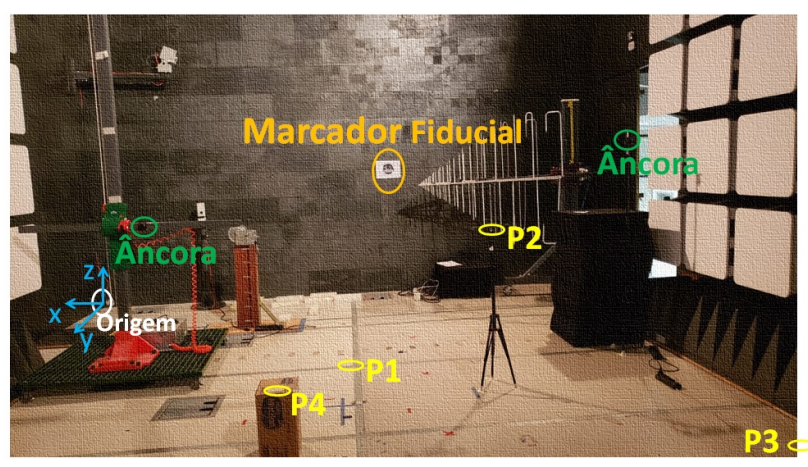

(b)

Figura 1: Câmara semianecóica, cenário dos ensaios: (a) Visão do fundo; (b) Visão frontal. Fonte: Autores.

A pesquisa foi divida em três etapas: a definiç̧ão da premissa, a seleção das tecnologias de localização indoor que atendessem a essa premissa, e finalmente a realização dos ensaios comparativos entre as tecnologias selecionadas.

Para a consecução da primeira etapa, a precisão sugerida para um cenário industrial foi levantada mediante discussões de um grupo multidisciplinar de pesquisadores e especialistas em manutenção industrial e em
RA. O grupo contou com profissionais de diversas áreas de atuação, como Física, Engenharia da Computação, Engenharia Elétrica e Matemática. Como resultado destas discussões, foi estabelecido como premissa que a precisão para localização de componentes deveria ser inferior ou igual a $10 \mathrm{~cm}$.

Na segunda etapa, foram selecionadas tecnologias de localização indoor que atendessem a essa premissa. Essa seleção levou em consideração métricas baseadas em uma revisão de literatura, na qual o foco foram as publicações dos últimos dois anos sobre o tema sistema de posicionamento indoor.

Na terceira etapa, de realização dos ensaios comparativos, foram adquiridos dispositivos de mercado capazes de realizar a medição de coordenadas tridimensionais com a precisão mínima de $10 \mathrm{~cm}$. Para testar e comparar cada tecnologia de localização selecionada na etapa anterior, ou seja, Ultra-wide-band (UWB), Sinal Acústico e Baseada em Imagens, foram adquiridos três dispositivos: Pozyx NV (2015), Robotics (2017) e Microsoft (2015), respectivamente.

Os ensaios utilizaram as ferramentas nativas disponibilizadas pelos próprios fabricantes dos três dispositivos adquiridos, respeitando as configurações e especificações sugeridas em documentação e seguindo as recomendações publicadas em sites e/ou fóruns oficiais. Os ensaios realizados para cada uma das tecnologias foram realizados de forma sequencial, em uma câmara semianecóica. Essa câmara foi escolhido para que houvesse o mínimo possível de interferências externas no comportamento dos dispositivos. A Fig. 1 mostra o ambiente físico onde foram realizados os ensaios, ilustrando uma visão da frente para o fundo da câmara (Fig. 1(a)) e do fundo para a frente (Fig. 1(a)). Os locais demarcados em amarelo ilustram a disposição física dos pontos avaliados dentro do cenário; em branco, observa-se o local utilizado como origem do sistema de coordenadas; em azul, verifica-se a direção dos eixos de coordenadas; em laranja, destaca-se o marcador fiducial utilizado pela tecnologia baseada em imagens; e, em verde, estão sinalizadas as posições física das âncoras utilizadas nas tecnologias UWB e Sinal Acústico. Neste trabalho o termo âncoras é adotado para designar dispositivos, com posição fixa, responsáveis por se comunicarem com os dispositivos móveis para rastreá-los dentro do ambiente.

Finalmente, adquiridos os três dispositivos de mercado para comparar cada tecnologia de localização selecionada e definida a câmara semianecóica como cenário dos testes, foram realizados dois ensaios, o primeiro de Análise de Precisão e Estabilidade, com objetivo de analisar o comportamento do sistema quanto a sua dispersão e precisão e o segundo de Análise de Propagação de Erros, com objetivo de observar a capacidade de o sistema evitar propagações de erros provocados pelo deslocamento dentro do ambiente de testes.

Na próxima seção são apresentados e discutidos os resultados obtidos nestas etapas. 


\section{Descrição dos ensaios e análise dos resul- tados}

As próximas subseções descrevem os resultados obtidos nas etapas de seleção das tecnologias de localização indoor a serem comparadas e dos dois ensaios realizados.

\subsection{Seleção das tecnologias de localização in- door a serem comparadas}

A revisão do estado da arte do conhecimento sobre o tema localização indoor apontou que diversos autores propõem diferentes critérios para comparação de IPS's. A seguir mostramos uma compilação dos critérios baseado nos trabalhos de Wang et al. (2017), Alarifi et al. (2016), Yassin et al. (2017):

i. Precisão/Acurácia - Distância média euclidiana entre uma posição estimada e a posição real (Liu et al., 2007).

ii. Privacidade do usuário - Controle de acesso estrito às informações pessoais dos indivíduos (Liu et al., 2007) (Mautz, 2012);

iii. Área de cobertura - Área de abrangência do IPS, incluindo três níveis;

\section{i. Local,}

ii. Escalável,

iii. Global (Mautz, 2012).

iv. Dependência de dispositivo do usuário - Necessidade de equipamento extra sendo transportado para o IPS funcionar;

v. Custo - Valor associado à utilização do IPS, que pode levar em consideração não só questões financeiras como também outros tipos de valores, por exemplo, instalação de infraestrutura, necessidade de manutenção, consumo de energia, tamanho dos dispositivos (custo de espaço) (Gu et al., 2009) (Nuaimi and Kamel, 2011);

vi. Complexidade - $\mathrm{O}$ enredo necessário para projetar, construir e manter um IPS, que dependendo do valor associado à avaliação do IPS, pode estar contida em Custos.

vii. Continuidade ou Disponibilidade - Capacidade de manter-se funcionando, de forma ininterrupta, em sua função específica;

viii. Taxa de atualização - Frequência de atualização das posições no IPS;

ix. Saída de dados - Forma como os dados são disponibilizados para outros sistemas;

$\mathrm{x}$. Eficiência energética - capacidade de manter-se funcionando pelo maior tempo possível;

Wang et al. (2017) avaliam as tecnologias de acordo com alguns dos critérios acima enumerados e sintetiza os resultados conforme Tabela 1, utilizada como referência para seleção das tecnologias a serem testadas

Considerando as especificações dos IPS's e a premissa de precisão desejada inferior ou igual a $10 \mathrm{~cm}$, identificou-se que, das tecnologias aplicáveis, apenas três atendem ao critério : UWB, Sinal Acústico (Acous- tic Signal), Baseada em Imagens (Image Based), Luz Visível (Visible Light) e Geomagnetismo. Esta última, embora esteja no limite da precisão almejada, foi descartada por sofrer forte influência de campos magnéticos, comuns em ambientes industriais. A tecnologia Luz Visível também foi descartada em virtude das interferências de luminosidade que normalmente ocorrem em cenários industriais.

Após exame das tecnologias de localização que atendem a premissa deste trabalho, foram adquiridos dispositivos de mercado capazes de realizar a medição de coordenadas tridimensionais com a precisão desejada para a realização dos ensaios.

\subsection{Realização dos ensaios comparativos}

\subsubsection{Ensaio 1: Análise de precisão e estabilidade}

$\mathrm{O}$ aspecto observado neste ensaio foi a precisão e estabilidade das coordenadas virtuais aferidas pelos dispositivos adquiridos.

Para realização do ensaio, primeiramente foi escolhido o canto da câmara semianecóica como origem para os sistemas de coordenadas reais e virtuais. Após isso, foram demarcados no cenário os cinco pontos (P1, P2, P3, P4 e P5), conforme demonstrado na Fig. 1 (a) e Fig. 1 (b), e, com auxílio de uma trena laser FLUKE $419 \mathrm{D}$, foram anotadas as coordenadas espaciais $(\mathrm{x}, \mathrm{y}, \mathrm{z})$ da localização de cada um dos cinco pontos. Além disso, foram demarcados e medidos também os quatro pontos onde ficariam posteriormente as âncoras de alguns dos dispositivos.

A fim de analisar as tecnologias de UWB e Sinal Acústico, as quatro âncoras que acompanham cada um dos kits Pozyx NV (2015) e Robotics (2017) foram posicionadas no ambiente de testes, na mesma posição, porém em momentos distintos para os ensaios, respeitando os requisitos definidos na documentação dos fornecedores. No ensaio de cada uma das tecnologias, foi realizada a calibração do sistemas de coordenadas virtuais. A calibração respeitou todos os requisitos disponíveis na documentação dos fornecedores dos dispositivos. Entretanto, é importante ressaltar que, diferentemente da calibração do Pozyx, na qual as coordenadas das âncoras são inseridas manualmente no sistema, na calibração do Marvelmind, o único parâmetro inserido manualmente é a coordenada do eixo $\mathrm{z}$. As coordenadas dos eixos x e y são medidas de forma automática mediante a comunicação entre as próprias âncoras.

Com o objetivo de realizar as medições das coordenadas virtuais, foi posicionada, na etapa de ensaio para cada tecnologia, o dispositivo móvel do kit em cada um dos cinco pontos medidos fisicamente. Para ambos os dispositivos, foram cronometrados 60 segundos para estabilização do sistema e, após isso, foram coletadas 60 segundos de amostras, referentes às coordenadas virtuais de cada um dos pontos.

Para a avaliação da tecnologia baseada em imagens, foi desenvolvido um aplicativo que utiliza os recursos do óculos Hololens para realizar a captura das coordenadas virtuais, a partir de um ponto de calibração. $O$ ponto de calibração foi demarcado com um marcador fiducial e foi processado na aplicação utilizando o framework 
Tabela 1: Comparação de IPS's. Fonte: Adaptada de Wang et al. (2017)

\begin{tabular}{|c|c|c|c|}
\hline Tecnologia & Precisão (m) & Vantagens & Desvantagens \\
\hline A-GPS & $5-10$ & $\begin{array}{l}\text { Sensores embarcados no celular; Cober- } \\
\text { tura mundial. }\end{array}$ & $\begin{array}{l}\text { Baixa segurança; Ocupa canal no celu- } \\
\text { lar. }\end{array}$ \\
\hline GSM (cellular) & $10-50$ & $\begin{array}{l}\text { Livre de interferências; Sensores embar- } \\
\text { cados no celular. }\end{array}$ & $\begin{array}{l}\text { Baixa confiabilidade; Questões de Priva- } \\
\text { cidade. }\end{array}$ \\
\hline RFID & $1-3$ & Custo moderado; Alta precisão. & $\begin{array}{l}\text { Não há comunicação entre tags; Cober- } \\
\text { tura limitada; Necessidade de disposi- } \\
\text { tivo externo. }\end{array}$ \\
\hline WiFi & $1-5$ & Utiliza infraestrutura do local. & Recálculo do algoritmo Fingerprinting; \\
\hline UWB & $0.06-0.1$ & $\begin{array}{l}\text { Excelente precisão; Sinal ultrapassa obs- } \\
\text { táculos. }\end{array}$ & $\begin{array}{l}\text { Alto custo; Baixa cobertura; Problemas } \\
\text { quando não há LoS. }\end{array}$ \\
\hline Dead Reckoning & $1-5$ & Só necessita de beacons & Baixa precisão. \\
\hline Infrared & $1-2$ & Baixo consumo de energia. & $\begin{array}{l}\text { Baixa cobertura; Despesas com hard- } \\
\text { ware extra. }\end{array}$ \\
\hline BLE Beacon & $1-5$ & Baixo custo e consumo de energia. & $\begin{array}{l}\text { Limitação de mobilidade do usuário; } \\
\text { Baixa precisão. }\end{array}$ \\
\hline Acoustic Signal & $0.03-0.8$ & $\begin{array}{l}\text { Não necessita LoS; Não sofre interferên- } \\
\text { cias eletromagnética. }\end{array}$ & $\begin{array}{l}\text { Não ultrapassa paredes sólidas; Perda } \\
\text { de sinal por obstrução; Reflexo de sinal. }\end{array}$ \\
\hline ZigBee & $1-10$ & $\begin{array}{l}\text { Baixo custo de infraestrutura e consumo } \\
\text { de energia; Alta frequência de atualiza- } \\
\text { ção e segurança; }\end{array}$ & Baixa cobertura. \\
\hline Visible Light & $0.01-0.1$ & $\begin{array}{l}\text { Uso duplo da infraestrutura; Compatível } \\
\text { com áreas sensíveis a rádio frequência. }\end{array}$ & $\begin{array}{l}\text { Necessidade de substituição da ilumina- } \\
\text { ção local; Custo elevado. }\end{array}$ \\
\hline Image-Based & $0.01-1$ & $\begin{array}{l}\text { Relativamente barato se comparado a } \\
\text { UWB. }\end{array}$ & $\begin{array}{l}\text { Necessidade de canal direto com o sinal; } \\
\text { Cobertura limitada. }\end{array}$ \\
\hline Geomagnetism & $0.10-2$ & $\begin{array}{l}\text { Não requer manutenção; Capacidade de } \\
\text { ultrapassar paredes. }\end{array}$ & $\begin{array}{l}\text { Interferência de campos magnéticos do } \\
\text { ambiente. }\end{array}$ \\
\hline
\end{tabular}

de Realidade Aumentada Vuforia (PTC Inc., 2018). Durante o ensaio, após a calibração do sistema através do marcador fiducial, deslocou-se do ponto de calibração para cada um dos cinco pontos do cenário. Em cada um dos pontos, foi posicionado manualmente o cursor do óculos, em seguida, foi cronometrado 60 segundos para estabilização do sistema e, após isso, foram coletadas mais 60 segundos de amostras, referentes às coordenadas virtuais dos respectivos pontos. A captura de coordenadas com o Hololens foi um processo manual em que um usuário vestia o óculos, iniciava a aplicação, posicionava com a cabeça o cursor, implementado na aplicação, no local demarcado com o ponto a ser medido e iniciava-se, com um clique, a coleta de amostras. Qualquer erro humano que porventura tenha acontecido durante a coleta das amostras serão devidamente comentados nos resultados.

A Fig. 2 resume as etapas realizadas no ensaio $1 \mathrm{e}$ os resultados obtidos serão discutidos no decorrer deste trabalho.

\subsubsection{Resultados e análises do Ensaio 1}

Com o intuito de facilitar a análise dos resultados, todas as coordenadas medidas, tanto com a trena quanto pelos sistemas de coordenadas virtuais, foram convertidas para centímetros. É importante ressaltar que todos os pontos escolhidos tinham linha direta com todas as âncoras.

A Fig. 1 ilustra a disposição real dos pontos, eixos, origem, âncoras e do marcador utilizados nos ensaios. Durante os 60 segundos de coleta para cada uma das tecnologias, para cada um dos pontos, o dispositivo Hololens gerou 3600 amostras das coordenadas, o Mar- velmind 500 e o Pozyx 200. Foram detectados alguns outliers extremos, ou seja, valores de dados que estavam a mais de 3,0 vezes a faixa interquartílica abaixo do primeiro quartil ou acima do terceiro quartil (Jones, 1996). Estes outliers extremos foram retirados da amostra e, para igualar a quantidade de dados, as amostras do Hololens e do Marvelmind foram selecionadas por amostragem aleatória.

A Fig. 3 mostra o gráfico da dispersão das amostras, sua média, mediana e precisão em todos os pontos. A precisão foi avaliada verificando-se se o vetor de distância formado entre o valor da coordenada real, medida com a trena, e o valor aferido pelas tecnologias era inferior a $10 \mathrm{~cm}$.

O primeiro ponto medido ( $\mathrm{P} 1)$ estava localizado no chão e em uma das extremidades da estrutura geométrica formada pelas âncoras. As coordenadas reais medidas no P1 para os eixos $x, y$ e $z$ foram, respectivamente, $-285 \mathrm{~cm}, 274 \mathrm{~cm}$ e $0 \mathrm{~cm}$. A Fig. 3 mostra os gráficos da dispersão dos dados coletados pelas três tecnologias no P1. É possível verificar que o Pozyx foi, dos três dispositivos, o que apresentou maior instabilidade. Das amostras coletadas do Hololens e do Marvelmind, todas tiveram uma diferença inferior a 10 $\mathrm{cm}$ da posição real. Já quanto ao Pozyx, todas as amostras foram superiores a $10 \mathrm{~cm}$ de distância do valor real. Um ponto importante a ser frisado é que, apesar do Marvelmind ter tido todos os seus pontos dentro dos $10 \mathrm{~cm}$ de precisão, houve uma grande dispersão dos dados.

O P2 foi escolhido para ser colocado em um tripé, mais ao centro do cenário, com $-387 \mathrm{~cm}$ na coordenada $x, 347 \mathrm{~cm}$ na $y$ e $111 \mathrm{~cm}$ na coordenada $z$. A Fig. $3 \mathrm{de}-$ 


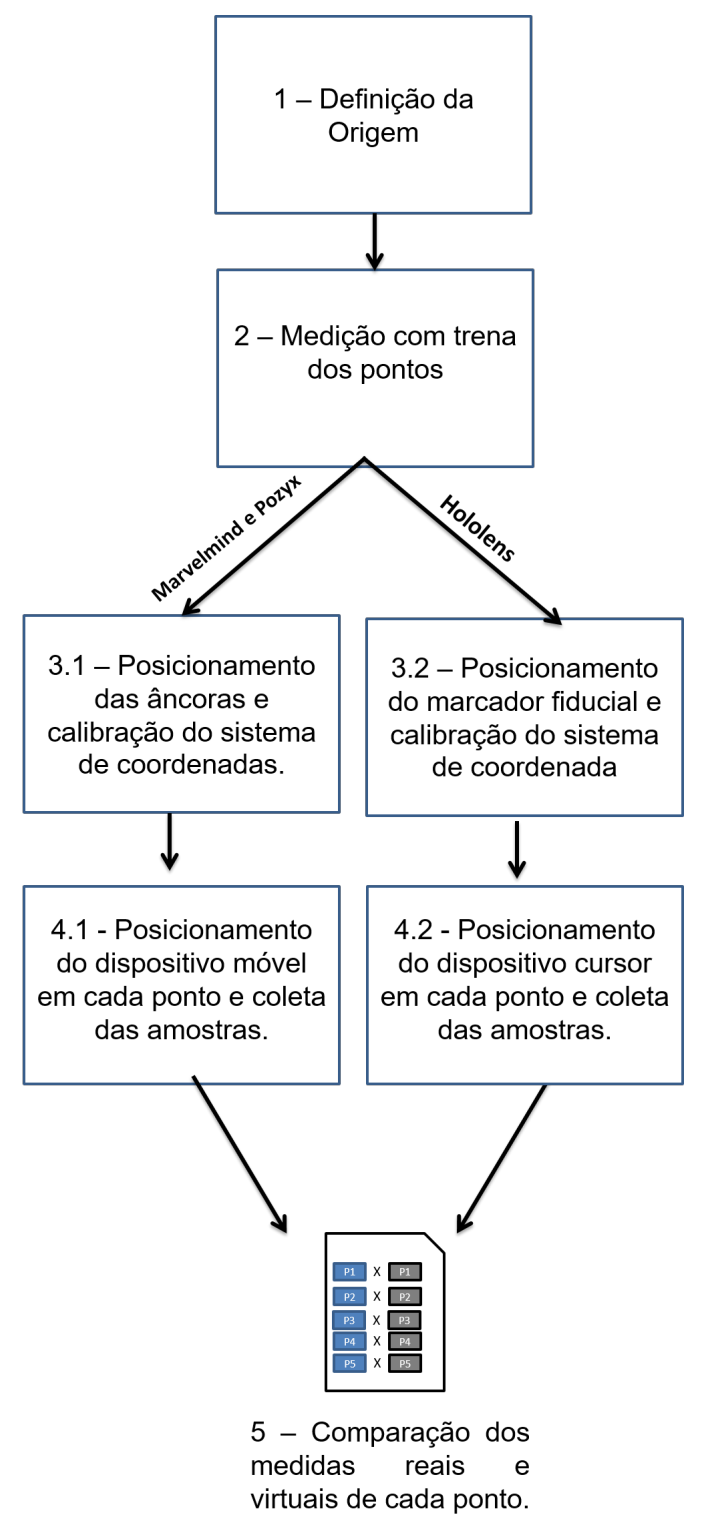

Figura 2: Ensaio 1: Análise de precisão e estabilidade. Fonte: Autores.

monstra a dispersão das amostras em cada uma das tecnologias testadas. Mais uma vez, todas as amostras do Hololens e do Marvelmind se mantiveram dentro de $10 \mathrm{~cm}$ de precisão e com desvio padrão em 0 . O Pozyx continuou com uma dispersão muito grande em todos os eixos, com $84 \%$ das amostras superiores a $10 \mathrm{~cm}$ de precisão. Não há nenhuma evidência aparente, nesse ponto, que justifique resultados fora do esperado para nenhuma das tecnologias.

As coordenadas reais do $\mathrm{P} 3$ são $-507 \mathrm{~cm}, 621 \mathrm{~cm}$ e $0 \mathrm{~cm}$ para $x, y$ e $z$. Esse ponto foi propositalmente colocado no chão e em outra extremidade formada por duas das âncoras. Na Fig. 3 é possível analisarmos o resultado obtido. Nesse ponto o Marvelmind obteve um resultado pouco satisfatório, onde todas as suas amostras tiveram imprecisão superior a $10 \mathrm{~cm}$, bem
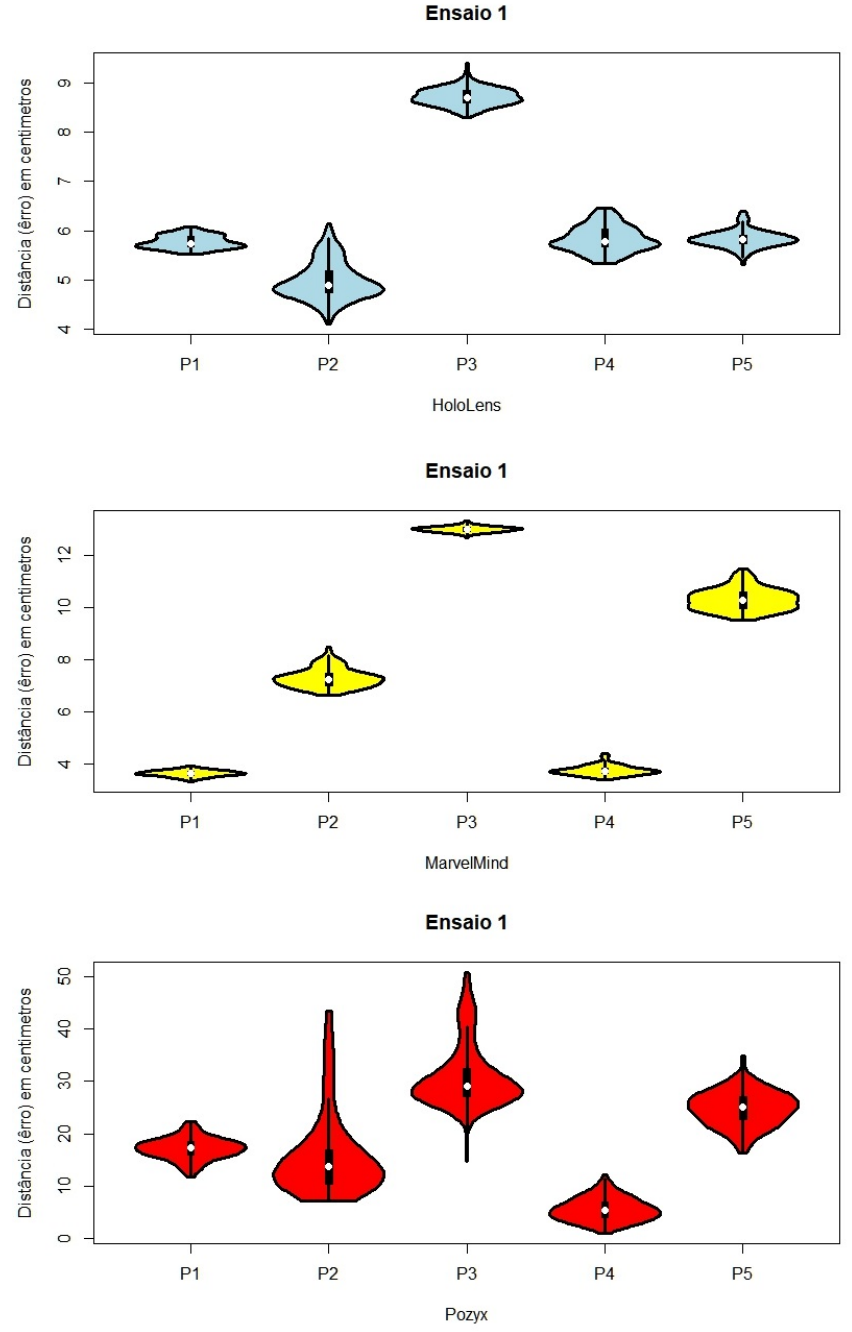

Figura 3: Resultados do Ensaio 1.

como o Pozyx que obteve $100 \%$ das amostras fora da margem de precisão. Já o Hololens continuou com bons resultados tanto no que diz respeito à estabilidade quanto à precisão, com todas as amostras dentro da precisão esperada.

$\mathrm{O}$ ponto $\mathrm{P} 4$ foi posicionado sobre uma caixa de papelão e ao centro do cenário, conforme pode ser observado na Fig. 1. As coordenadas foram $-248 \mathrm{~cm}, 425 \mathrm{~cm} \mathrm{e}$ $38 \mathrm{~cm}$. Os gráficos da Fig. 3 demonstram o comportamento dos sistemas. Nesse ponto, todas as tecnologias obtiveram bons resultados. O Pozyx foi a única que obteve apenas $3 \%$ das amostras fora da precisão de $10 \mathrm{~cm}$. Todas as demais amostras foram dentro da margem de precisão.

$\mathrm{O}$ ponto $\mathrm{P} 5$ foi colocado no chão e posicionado mais ao centro do cenário (Fig. 1). As suas coordenadas em $x$, $y$ e $z$ são: $-198,591$ e 0 . Nos gráficos representativos da dispersão das amostras, é possível observar que, exceto o Hololens que obteve todas as suas amostras precisas, o Marvelmind obteve $74 \%$ das amostras imprecisas e o Pozyz $100 \%$ das amostras maiores do que $10 \mathrm{~cm}$ de 
Tabela 2: Proporção de resultados para cada equipamento, contidos dentro da margem de erro de 10 centímetros de distância.

\begin{tabular}{cccccc}
\hline \hline & P1 & P2 & P3 & P4 & P5 \\
\hline HOLOLENS & 100 & 100 & 100 & 100 & 100 \\
MARVELMIND & 100 & 100 & 0 & 100 & 26 \\
POZYX & 0 & 16 & 0 & 97 & 0
\end{tabular}

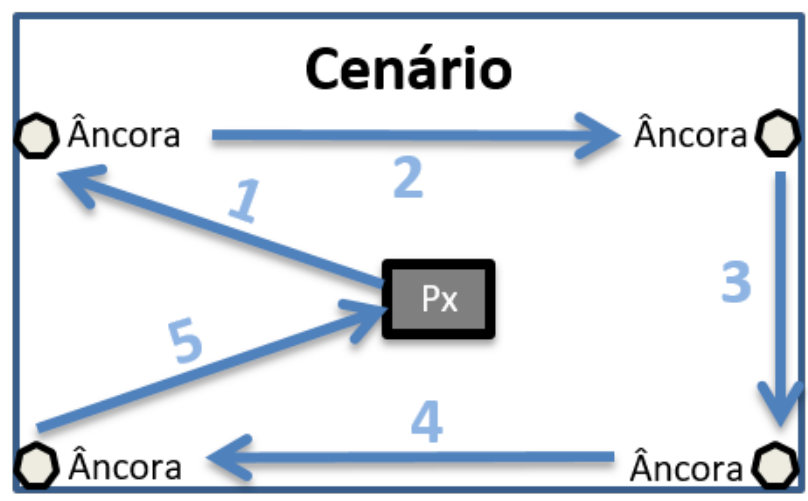

Figura 4: Percurso fixo realizado no Ensaio 2.

distância do ponto real.

A Tabela 2 condensa a precisão obtida pelos três dispositivos. Nela é possível observar que o Hololens obteve um resultado muito bom, com $100 \%$ das amostras dentro da precisão esperada. O Marvelmind obteve $34,8 \%$ dos pontos fora do intervalo de 0 a $10 \mathrm{~cm}$ de precisão, sendo que seu pior ponto foi o P3, com $100 \%$ das amostras imprecisas. Por fim, o Pozyx das três tecnologias foi o que não apresentou bons resultados, com apenas $22,6 \%$ das amostras dentro da margem de $10 \mathrm{~cm}$ de precisão.

\subsubsection{Ensaio 2: Análise de Propagação de Erros}

$\mathrm{O}$ aspecto observado neste ensaio foi a capacidade do sistema evitar propagações de erros provocados pelo deslocamento dentro do ambiente de testes.

Para a realização desse ensaio foi escolhido o ponto onde todas as tecnologias obtiveram bons resultados no ensaio 1, o ponto P4, conforme pode ser observado sua localização na Fig. 1. O procedimento seguido nesse ensaio, para cada uma das tecnologias, foi o mesmo seguido no ensaio anterior até a etapa de calibração dos sistemas. Após calibrado, utilizando os IPS Pozyx, Marvelmind e Hololens (este com o aplicativo desenvolvido para o presente trabalho), em momentos distintos e para cada um dos dispositivos foram cronometrados 60 segundos para estabilização dos sistemas e, logo após isso, foram coletados 60 segundos de amostras referentes às coordenadas virtuais do ponto a ser avaliado. Após cada uma das medições e sem desligar os dispositivos, era feito um percurso fixo, pré-definido (Fig. 4), ao redor do ambiente de testes, retornando-se em seguida à mesma posição. $O$ percurso foi realizado nove vezes para cada uma das tecnologias testadas.

Como a frequência de geração das amostras é dife-

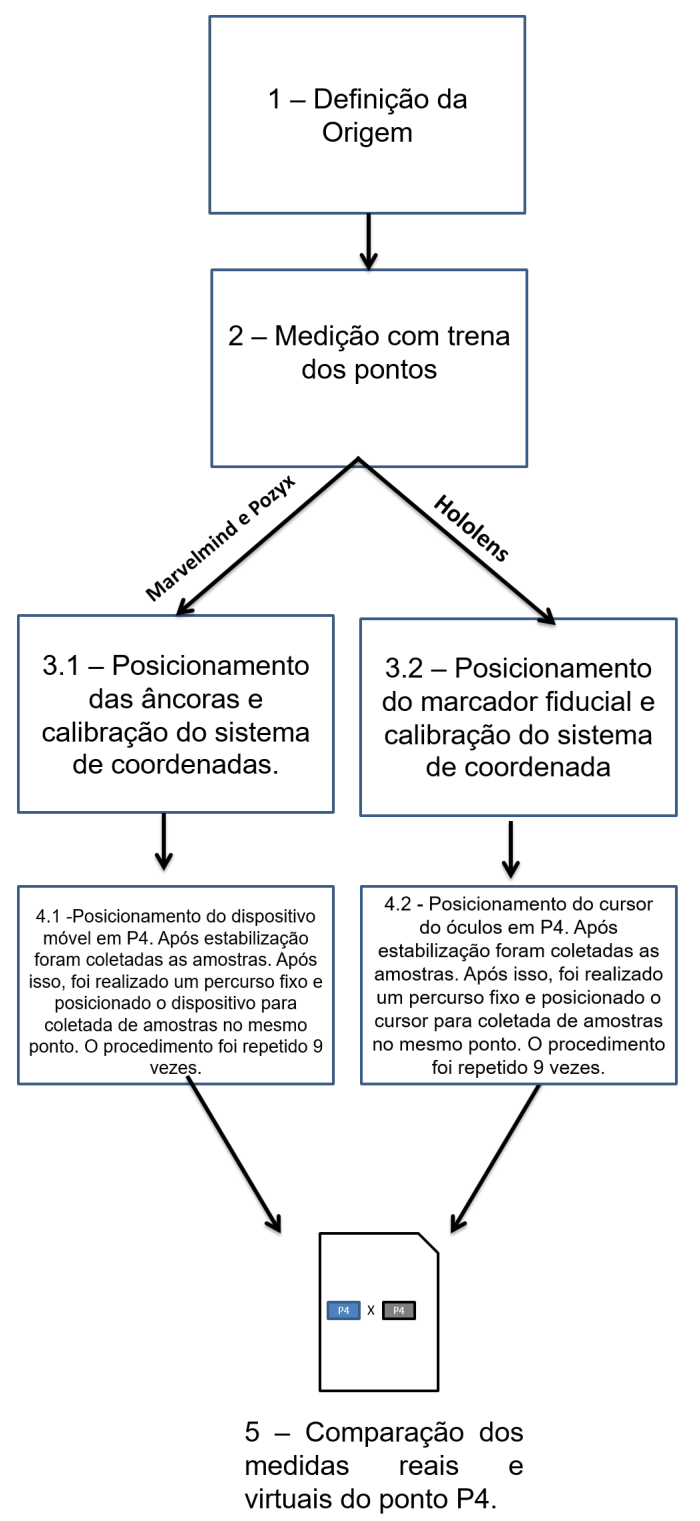

Figura 5: Ensaio 2: Análise de Propagação de Erros. Fonte: Autores.

rente para cada uma das tecnologias, o Pozyx gerou 2000, o Marvelmind 5000 e o Hololens 36000 . Seguindo o mesmo procedimento do ensaio anterior, para análise dos resultados, foram desconsiderados os outliers extremos. A Fig. 5 resume o procedimento realizado e novamente os resultados obtidos serão discutidos no decorrer deste trabalho.

\subsubsection{Resultados e análises do Ensaio 2}

Os resultados obtidos nesse ensaio estão resumidos nos gráficos da Fig. 6. É possível observar que as tecnologias avaliadas não aumentaram a imprecisão no decorrer dos ensaios, ou seja, elas se mantiveram constantes na sua variação das amostras.

Tanto o Hololens quanto o Marvelmind mantiveram todas as suas amostras dentro dos 10 centímetros de 


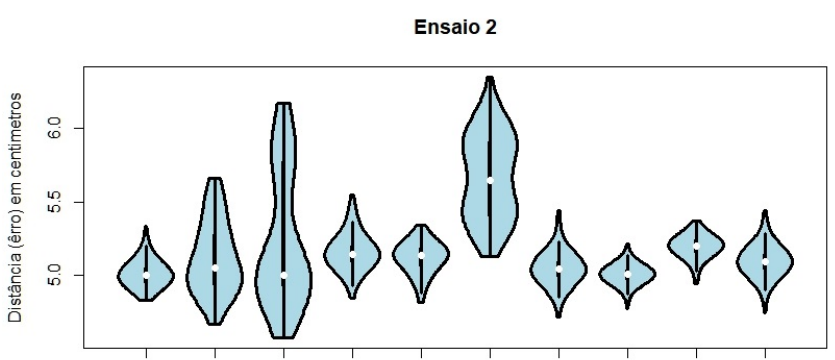

Inicial Volta 1 Volta 2 Volta 3 Volta 4 Volta 5 Volta 6 Volta 7 Volta 8 Volta 9

HoloLens

Ensaio 2

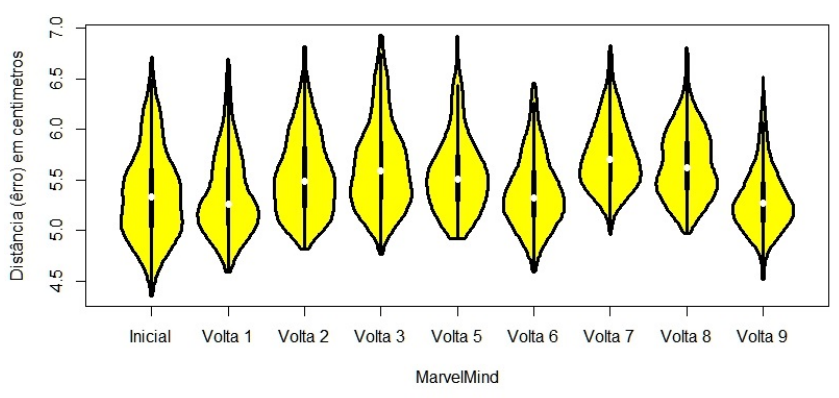

Ensaio 2

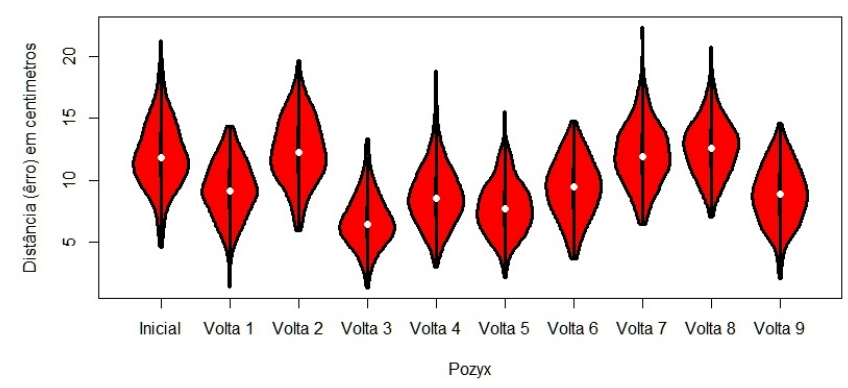

Figura 6: Resultados do Ensaio 2.

precisão. Somente o Pozyx obteve amostras fora do intervalo de precisão. É importante ressaltar que, na volta 04 do Marvelmind, após 11 segundos de coleta, os valores das coordenadas passaram a ser constantes em $x=-250, y=427$ e $z=43$. Essa informação sugere um travamento do sistema, entretanto, para todas as voltas de todos os dispositivos testados, o sistema não era desligado e nem reiniciado. Presume-se que o sistema tenha voltado ao seu comportamento normal após a retirada do dispositivo do ponto e a realização da volta subsequente.

\section{Conclusão}

Este trabalho teve como objetivo avaliar a imprecisão gerada por dispositivos de localização indoor na medição de coordenadas virtuais, para futura aplicação em RA no contexto industrial. Em outras palavras, este trabalho avaliou, dentre os sistemas de posicionamento indoor aplicáveis, qual alcançou a maior precisão na lo- calização de coordenadas tridimensionais, de forma a ser integrada com RA para a indústria.

Os resultados dos dois ensaios comparativos, de análise de precisão e estabilidade e de análise de propagação de erros, permitiram avaliar as tecnologias de localização indoor que apresentaram o melhor desempenho quanto à precisão.

Das três tecnologias comparadas, as que obtiveram melhores resultados para a localização em ambientes indoor foram as tecnologias baseadas em imagens, através do dispositivo Hololens e a de Ultrassom, avaliada por meio do dispositivo Marvelmind. Com relação ao Hololens, é importante ressaltar que, mesmo com a possibilidade de erro humano, devido ao balanço da cabeça, em todos os ensaios realizados este sempre manteve-se muito estável e preciso. Já o Pozyx, apresentou uma dispersão muito grande das suas amostras, o que demonstra muita instabilidade, mesmo estando em uma câmara semianecôica que bloqueia boa parte das interferências externas. Por fim, o Marvelmind com a tecnologia de Ultrassom se mostrou estável e preciso na maior parte das vezes, demonstrando resultados ruins em apenas dois dos pontos testado, o $\mathrm{P} 3 \mathrm{e}$ o P5.

Todas as tecnologias demonstraram que não propagaram o erro com o decorrer dos ensaios. Elas sempre se mantiveram dentro da sua margem de variação, não havendo uma elevação constante dos pontos da amostra.

A partir dos resultados dos ensaios comparativos, é possível afirmar que a tecnologia do Hololens alcançou a melhor precisão dentre as tecnologias testadas.

Este trabalho comparou dispositivos de mercado que representam as três tecnologias selecionadas: UWB, ultrassom e baseada em imagens. Ou seja, foram eleitos dispositivos de mercado capazes de representar as tecnologias testadas. Como trabalho futuro, sugere-se realizar os mesmos ensaios, com outros dispositivos comerciais baseados nestas tecnologias, para corroborar os resultados.

\section{Referências}

Alarifi, A., Al-Salman, A., Alsaleh, M., Alnafessah, A., Al-Hadhrami, S., Al-Ammar, M. A. and Al-Khalifa, H. S. (2016). Ultra wideband indoor positioning technologies: Analysis and recent advances, Sensors 16(5). https://doi.org/10.3390/s16050707.

Azuma, R., Baillot, Y., Behringer, R., Feiner, S., Julier, S. and MacIntyre, B. (2001). Recent advances in augmented reality, IEEE Comput. Graph. Appl. 21(6): 34-47. https://doi.org/10.1109/38.963459.

Azuma, R. T. (1997). A survey of augmented reality, Presence: Teleoper. Virtual Environ. 6(4): 355-385. http: //dx.doi.org/10.1162/pres.1997.6.4.355.

Carmigniani, J. and Furht, B. (2011). Augmented reality: An overview, in B. Furht (ed.), Handbook of Augmented Reality, Springer New York, New York, NY, pp. 3-46. https://doi.org/10.1007/978-1-4614-0064-6_1. 
Comport, A. I., Marchand, E., Pressigout, M. and Chaumette, F. (2006). Real-time markerless tracking for augmented reality: the virtual visual servoing framework, IEEE Transactions on Visualization and Computer Graphics 12(4): 615-628. https : //doi . org/10.1109/ TVCG.2006.78.

Correa, A., Barcelo Llado, M., Morell, A. and Vicario, J. L. (2016). Indoor pedestrian tracking by on-body multiple receivers, IEEE Sensors Journal 16(8): 25452553. https://doi.org/10.1109/JSEN. 2016.2518872.

Glockner, H., Jannek, K., Mahn, J. and Theis, B. (2014). Augmented Reality in Logistics, DHL Customer Solutions and Innovation.

Gu, Y., Lo, A. and Niemegeers, I. (2009). A survey of indoor positioning systems for wireless personal networks, Commun. Surveys Tuts. 11(1): 13-32. https: //doi.org/10.1109/SURV.2009.090103.

Jones, J. (1996). Math 170 - introduction to statistics. Disponível em: https://people.richland.edu/james/ lecture/m170/.

Lee, S., Ma, Y.-S., Thimm, G. and Verstraeten, J. (2008). Product lifecycle management in aviation maintenance, repair and overhaul, Computers in Industry 59(2): 296 - 303. https://doi.org/10.1016/j. compind.2007.06.022.

Liu, H., Darabi, H., Banerjee, P. and Liu, J. (2007). Survey of wireless indoor positioning techniques and systems, Trans. Sys. Man Cyber Part C 37(6): 1067-1080. http://dx.doi.org/10.1109/TSMCC. 2007.905750.

Martinetti, A., Marques, H. C., Singh, S. and van Dongen, L. (2019). Reflections on the limited pervasiveness of augmented reality in industrial sectors, Applied Sciences 9(16): 3382. http://dx. doi .org/10.3390/ app9163382.

Mautz, R. (2012). Indoor positioning technologies, Habilitation thesis, Department of Civil, Environmental and Geomatic Engineering, Institute of Geodesy and Photogrammetry. https://doi.org/10.3929/ ethz-a-007313554.

Microsoft (2015). Hololens. Disponível em https : //www . microsoft.com/en-us/hololens/.

Neges, M., Koch, C., König, M. and Abramovici, M. (2017). Combining visual natural markers and imu for improved ar based indoor navigation, Advanced Engineering Informatics 31: 18 - 31. https://doi.org/ 10.1016/j.aei.2015.10.005.

Nuaimi, K. A. and Kamel, H. (2011). A survey of indoor positioning systems and algorithms, 2011 International Conference on Innovations in Information Technology, pp. 185-190. https://doi.org/10.1109/INNOVATIONS. 2011.5893813.

Ong, S., Yuan, M. and Nee, A. (2008). Augmented reality applications in manufacturing: a survey, International Journal of Production Research 46(10): 2707-2742. https://doi.org/10.1080/00207540601064773.
Palmarini, R., Erkoyuncu, J. A., Roy, R. and Torabmostaedi, H. (2018). A systematic review of augmented reality applications in maintenance, Robotics and Computer-Integrated Manufacturing 49(Supplement C): 215 - 228. https://doi.org/10.1016/j.rcim. 2017.06 .002 .

Pozyx NV (2015). Pozyx. Disponível em https://www . pozyx.io/.

PTC Inc. (2018). Vuforia engine developer portal. Disponível em https://developer .vuforia.com/.

Rankohi, S. and Waugh, L. (2013). Review and analysis of augmented reality literature for construction industry, Visualization in Engineering 1(1): 9. https: //doi.org/10.1186/2213-7459-1-9.

Robotics, M. (2017). Marvelmind. Disponível em https: //marvelmind.com/.

Sanna, A. and Manuri, F. (2016). A survey on applications of augmented reality, Advances in Computer Science: an International Journal 5(1): 18-27. Disponível em http://www.acsij.org/acsij/article/view/400.

Wang, Z., Yang, Z. and Dong, T. (2017). A review of wearable technologies for elderly care that can accurately track indoor position, recognize physical activities and monitor vital signs in real time, Sensors 17(2). https://doi.org/10.3390/s17020341.

Yassin, A., Nasser, Y., Awad, M., Al-Dubai, A., Liu, R., Yuen, C., Raulefs, R. and Aboutanios, E. (2017). Recent advances in indoor localization: A survey on theoretical approaches and applications, IEEE Communications Surveys Tutorials 19(2): 1327-1346. http: //dx.doi.org/10.1109/COMST. 2016.2632427.

Zhou, F., Duh, H. B.-L. and Billinghurst, M. (2008). Trends in augmented reality tracking, interaction and display: A review of ten years of ismar, Proceedings of the 7th IEEE/ACM International Symposium on Mixed and Augmented Reality, ISMAR '08, IEEE Computer Society, USA, p. 193-202. https://doi.org/10.1109/ ISMAR . 2008. 4637362. 